$\underline{\beta}$

\title{
Pharmacological effect of synobiotic and phytobiotic in kidney function and some blood parameters of chicken
}

\author{
Marwa Kamel O. I. ${ }^{1}$, Ashraf A. A. El-Komy ${ }^{2}$, Enas A. A. Farag ${ }^{2}$ \\ ${ }^{1}$ Animal Health Researches Institute, Benha Branch \\ ${ }^{2}$ Department of pharmacology, faculty of Veterinary Medicine, Benha University, Egypt \\ *Corresponding author E-mail: d.marwakamel@gmail.com
}

\begin{abstract}
The aim of this research was to study the effects of dietary supplementation of synbiotic, phytobiotics and their mixture on kidney functions, some blood parameters and histological criteria of broilers. A total of 200 broiler chicks were randomly chosen and divided into eight groups ( 25 birds in each). The first four groups were unvaccinated chicken which received a basal diet (group 1) or the same diet supplemented with $1 \mathrm{~g}$ synobiotic / kg body weight (group 2), $1 \mathrm{~g}$ phytobiotic /kg body weight (group 3) and $1 \mathrm{~g}$ synobiotic plus $1 \mathrm{~g}$ phytobiotic/kg body weight (group 4). The second four groups were vaccinated chicken and fed the same previous treatments with groups 5, 6, 7 and 8 , respectively. Therapeutic dose of synobiotic and phytobiotic or their mixture $(1 \mathrm{ml} / \mathrm{kg}$. body weight) orally for 3 successive days tended to decrease uric acid and creatinine in unvaccinated and vaccinated chicken at 7, 14 and 21 day. In this study uric acid and creatinine levels resulted in decrease, showing a significant improvement of kidney functions . Some different parameters of blood and histological criteria showed normal measurements with no adverse effect of treatments.
\end{abstract}

Keywords: Synobiotic; Phytobiotic; Vaccinated; Unvaccinated; Chicken.

\section{Introduction}

Feed additives are used in animals for a number of different reasons: effective as antibiotic to improve the health, performance of broiler chickens, growth promotion and increase immune response of chickens . ( Murarolli et al., 2014) .

Synbiotic Is defined as a mixture of probiotic and prebiotic that beneficially affect the host by: improving the survival and activity of beneficial microorganisms in the gut.( Awad et al., 2008).

Phytobiotics can be defined as plant derived products added to feed to improve performance. They were originated from leaves, roots, tubers or fruits of herbs, spices and other plants. They may be available in solid, dried, and ground forms, or as extracts (essential oils). In simple terms, phytobiotics are products of plant origin, and preparations such as thyme, oregano, turmeric and garlic are gaining interest among researchers and poultry producers.

Phytobiotic or phytogenic as feed additive used as pharmaceuticals in alternative medicine and as a natural therapy (Amad et al, 2011). Both plant extracts and essential oils have been reported to have antimicrobial properties against a wide range of pathogenic microbial organism in vitro and in vivo (Erdogan et al. 2010). Effects of the Dietary Inclusion of a Probiotic, a Prebiotic or their Combinations on the Growth Performance of Broiler Chickens were studied by Alaeldein et al ( 2015).

The aim of this experiment was to study the effect of synobiotic and phytobiotic or their mixture on kidney function, some blood parameters and histological criteria with unvaccinated and vaccinated chicken.

\section{Materials and methods}

\subsection{Drugs}

\subsubsection{Synobiotic}

a) Lactulose (lactulose 67\%)

This medication is a laxative used to treat constipation. It may help to increase the number of bowel movements per day and the number of days you have a bowel movement. Lactulose is a colonic acidifier that works by increasing stool water content and softening the stool.

b) lacteol fort (Lactobacillus LB) 6 Sachets

Composition: Each Sachet contains: Lactobacillus LB, Corresponding to lactobacillus delbruekii and lactobacillus fermentum. Treatment of acute and chronic diarrhea. 


\subsubsection{Phytobiotic}

a) Ropadiar $20 \%$

Composition: carvacrol (oregano oil), thymol (oregano oil), propylene glycol, polysorbate $80 \mathrm{E} 433$, alpha tocopherol (Vit.E). It was used as 1-2 ml per 10 litter water for 3 days. The product was used to improve productive efficiency for broiler chicken.

\subsection{Vaccine are used for increase immune responses against the most economically}

Important chicken diseases (new castle vaccine)

\subsection{Instrument}

1) Neubour's haemocytometer (for RBCs and WBCs count).

2) Centrifuge (MLWT 52-1 Germany) was used for separating serum.

3) Wintrobe haematocrit tubes for packed cell volume determination.

4) Deep freeze (-20 co) (Sital co., A.R.E.) was used for keeping serum samples.

5) Microtome (American Optical Company, U.S.A) was used for sectioning the tissues for histopathological studies.

6) 6-Ordinary microscope (Car I- Zeiss 0.65 ) was used for histopathological examinations

7) Other equipments: beakers, bottles, cotton, flasks, forcipes, gauze, graduated cylinders, , scissors, and syringes were used.

\subsection{Chicks and experimental design}

200 chicks (one day old) were randomly chosen and divided into 8 groups, each group contains :

Group (1): 25 chicks were served as normal non treated group considered as control group. Group(2) : 25 chicks of 14 day ages non vaccinated with ND were administrated $1 \mathrm{ml}$ synobiotic / kg body weigh orally for 3 days. Group (3) : 25 chicks of 14 day ages non vaccinated with ND were administrated $1 \mathrm{ml}$ phytobiotic $/ \mathrm{kg}$ body weigh orally for 3 days .Group ( 4$): 25$ chicks of 14 day ages non vaccinated with ND were administrated $1 \mathrm{ml}$ mix of synobiotic and phytobiotic /Kg body weight for 3 days Group (5) : 25 chicks were served as normal vaccinated group considered as control group. Group (6): 25 chicks of 14 day ages vaccinated with ND were administrated $1 \mathrm{ml}$ synobiotic $/ \mathrm{kg}$ body weight orally for 3 days. Group (7) : 25 chicks of 14 day ages vaccinated with ND were administrated 1 $\mathrm{ml}$ phytobiotic $/ \mathrm{kg}$ body weight orally for 3 days and Group ( 8) : 25 chicks of 14 day ages vaccinated with ND were administrated $1 \mathrm{ml}$ mix of synobiotic and phytobiotic $/ \mathrm{Kg}$ body weight for 3 days

\subsection{Sampling}

Blood samples were taken at the end of $7^{\text {th }}$ day, $14^{\text {th }}$ day and $21^{\text {th }}$ post- treatment in all groups. Five birds of each group were sacrificed for collection of blood samples. Two blood samples were taken from each group for biochemical and hematological studies. The first blood sample was collected without anticoagulant for separation of clear serum for biochemical analysis. These serum samples were used for biochemical analysis to determine some blood parameters, uric acid and creatinine concentration. The second sample of blood was collected in test tube mixed with heparin as anticoagulant. The sample was shacked several times to ensure mixing of blood with anticoagulant. These blood samples were used for hematological studies to determine erythrocytic count and total leucocytic count according to Natt and Herrick (1952). Hemoglobin concentration was determined according to Wintrobe (1967), while packed cell volume was determined as described by Cohen (1967).

\subsection{Serum biochemical analysis}

Blood samples which taken at the end of $7^{\text {th }}, 14^{\text {th }}$ and $21^{\text {th }}$ day and collected without anticoagulant were used to determine some blood parameters. Serum uric acid was determined according to White et al. (1970), while serum creatinine was determine as described by Foline (1934)

\subsection{Hematological studies}

Hemoglobin concentration, erythrocyte count and white blood cells were counted by using automatic blood cell counter (model HA-VET CLINDIAG).

\subsection{Histopathology study}

The treated chicks were sacrificed at $7^{\text {th }}, 14^{\text {th }}$ and $21^{\text {th }}$ days post treatment. Specimens were collected from liver, thymus, bursa and kidney from each sacrificed tested chicks and fixed directly in formalin $10 \%$. The fixed tissues in formal were processed by dehydration in different concentrations of ethanol ,cleared with xylol and embedded in paraffin, serial sections of 4-5 microns, which were stained with Hematoxylin and Eosin ( Banchroft and Gamble, 2008 ) for histopathology examinations.

\section{Statistical analysis}

Statistical analysis was conducted with the Statistical Package for Social Science (SPSS 16 Inc. Released, 2009) to determine if variables differed among groups. Comparison among means was conducted by one-way ANOVA and subsequent Duncan (1955), Probability values of less than $5 \%(\mathrm{p} \leq 0.05)$ were considered significant 


\section{Results}

\subsection{Effect on kidney function}

\subsubsection{Effect on serum uric acid}

Table (1) and figure (1) showed the effect of administration synobiotic and phyobiotic and mixture of them in unvaccinated and vaccinated chicken. Demonstrated therapeutic dose of synobiotic and phyobiotic ( $1 \mathrm{ml} / \mathrm{kg}$ body weight) orally for 3 days showed decrease in uric acid level at (7day, 14 day ,21day) compared to control group with unvaccinated chicken and significant decrease in uric acid level at 14 and 21 day in vaccinated chicken. Phytobiotic additive decrease uric acid concentration in both unvaccinated and vaccinated chicken during all period compared to control group .Mixture of synobiotic and phytobiotic showed significant decrease in uric acid level with unvaccinated and vaccinated chicken during the successive periods.

\subsubsection{Effect on serum creatinine}

Effect of administration synobiotic and phyobiotic and mixture of them in unvaccinated and vaccinated chicken are shown in Table(2) and figure(2). Therapeutic dose of synobiotic and phyobiotic ( $1 \mathrm{ml} / \mathrm{kg}$ body weight) were orally for 3 days. Demonstrated synobiotic $(1 \mathrm{ml} / \mathrm{kg}$ body weight) in unvaccinated and vaccinated chicken showed decrease of creatinine level at (7day, 14 day and 21 day), but the creatinine level at 14 and 21 day with vaccinated chicken was significant decrease compared to control group . Administration of phytobiotic ( $1 \mathrm{ml} / \mathrm{kg}$ body weight) in non vaccinated chicken showed decrease of creatinine level at (7day, 14 day ,21day) compared to control group and significant decrease in vaccinated chicken at the same periods. Mixture of synobiotic and phytobiotic showed significant decrease in creatinine level with unvaccinated and vaccinated chicken during all periods.

\begin{tabular}{|c|c|c|c|c|}
\hline \multirow{2}{*}{ Groups } & & \multicolumn{3}{|c|}{ Period after (days) } \\
\hline & & 7 & 14 & 21 \\
\hline \multirow{6}{*}{ 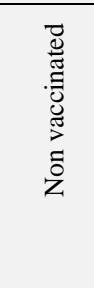 } & Control (-ve) & $9.39 \pm 0.18^{\mathrm{a}}$ & $9.39 \pm 0.46^{\mathrm{a}}$ & $9.49 \pm 0.39^{\mathrm{a}}$ \\
\hline & $\begin{array}{l}\text { Synobiotic } \\
(1 \mathrm{ml} / \mathrm{kg})\end{array}$ & $9.25 \pm 0.42^{\mathrm{ab}}$ & $8.96 \pm 0.10^{\mathrm{ab}}$ & $8.63 \pm 0.36^{\mathrm{ab}}$ \\
\hline & $\begin{array}{l}\text { Phytobiotic } \\
(1 \mathrm{ml} / \mathrm{kg})\end{array}$ & $9.17 \pm 0.20^{\mathrm{ab}}$ & $8.98 \pm 0.14^{\mathrm{ab}}$ & $8.48 \pm 0.33^{b}$ \\
\hline & Synobiotic + hytobiotic $(1 \mathrm{ml} / \mathrm{Kg})$ & $8.37 \pm 0.3^{\mathrm{b}}$ & $8.41 \pm 0.29^{\mathrm{b}}$ & $8.13 \pm 0.41^{\mathrm{b}}$ \\
\hline & Control (+ve) & $9.48 \pm 0.32^{\mathrm{a}}$ & $9.26 \pm 0.22^{\mathrm{a}}$ & $9.69 \pm 0.27^{\mathrm{a}}$ \\
\hline & $\begin{array}{l}\text { Synobiotic } \\
(1 \mathrm{ml} / \mathrm{kg})\end{array}$ & $8.81 \pm 0.34^{\mathrm{abc}}$ & $8.31 \pm 0.23^{\mathrm{b}}$ & $8.15 \pm 0.25^{\mathrm{bc}}$ \\
\hline \multirow{2}{*}{ 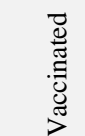 } & $\begin{array}{l}\text { Phytobiotic } \\
(1 \mathrm{ml} / \mathrm{kg})\end{array}$ & $8.17 \pm 0.23^{\mathrm{cd}}$ & $8.54 \pm 0.27^{b}$ & $7.92 \pm 0.17^{\mathrm{bc}}$ \\
\hline & $\begin{array}{l}\text { Synobiotic + hytobiotic } \\
(1 \mathrm{ml} / \mathrm{Kg})\end{array}$ & $7.63 \pm 0.25^{\mathrm{d}}$ & $7.39 \pm 0.23^{\mathrm{c}}$ & $7.30 \pm 0.37^{\mathrm{c}}$ \\
\hline
\end{tabular}

$\mathrm{a}, \mathrm{b} \& \mathrm{c}$ : There is no significant difference $(\mathrm{P}>0.05)$ between any two means, within the same column have the same superscript letter.

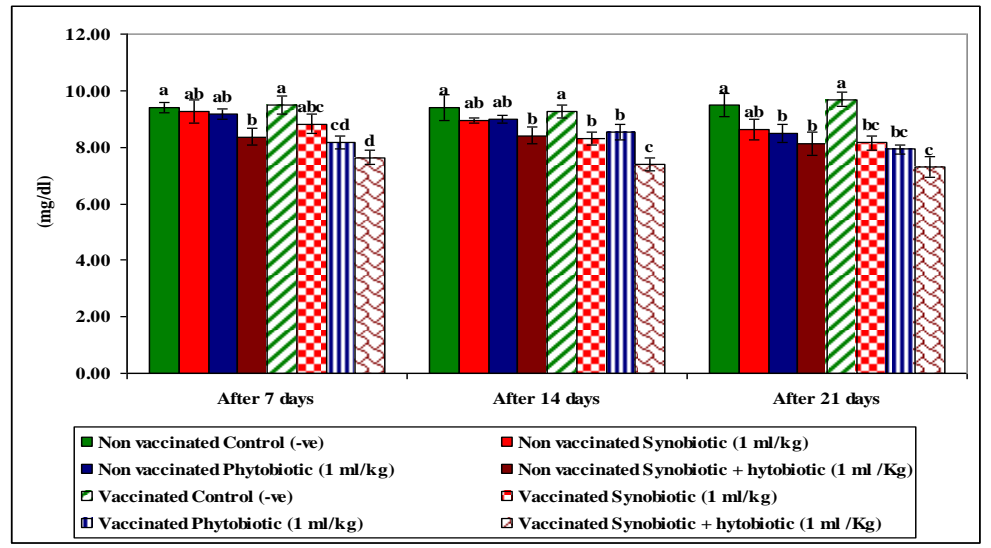

Fig. 1: Uric acid.

Table 2: Creatinine (Mg/Dl) (Mean $\pm \mathrm{SE})$

\begin{tabular}{|c|c|c|c|c|}
\hline \multirow{2}{*}{\multicolumn{2}{|c|}{ Groups }} & \multicolumn{3}{|l|}{ Period after (days) } \\
\hline & & & 14 & 21 \\
\hline \multirow{5}{*}{ 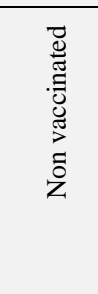 } & Control (-ve) & $0.33 \pm 0.01^{\mathrm{a}}$ & $0.32 \pm 0.01^{\mathrm{ab}}$ & $0.31 \pm 0.01^{\mathrm{ab}}$ \\
\hline & $\begin{array}{l}\text { Synobiotic } \\
(1 \mathrm{ml} / \mathrm{kg})\end{array}$ & $0.31 \pm 0.02^{\mathrm{ab}}$ & $0.31 \pm 0.01^{\mathrm{abc}}$ & $0.32 \pm 0.02^{\mathrm{a}}$ \\
\hline & $\begin{array}{l}\text { Phytobiotic } \\
(1 \mathrm{ml} / \mathrm{kg})\end{array}$ & $0.31 \pm 0.01^{\mathrm{ab}}$ & $0.33 \pm 0.02^{\mathrm{a}}$ & $0.32 \pm 0.02^{\mathrm{a}}$ \\
\hline & $\begin{array}{l}\text { Synobiotic + hytobiotic }(1 \mathrm{ml} / \mathrm{Kg}) \\
\text { Control (+ve) }\end{array}$ & $\begin{array}{l}0.29 \pm 0.02^{\mathrm{ab}} \\
0.33 \pm 0.01^{\mathrm{a}}\end{array}$ & $\begin{array}{l}0.28 \pm 0.01^{\mathrm{bc}} \\
0.30 \pm 0.01^{\mathrm{abc}}\end{array}$ & $\begin{array}{l}0.27 \pm 0.02^{\mathrm{bc}} \\
0.32 \pm 0.01^{\mathrm{a}}\end{array}$ \\
\hline & $\begin{array}{l}\text { Synobiotic } \\
(1 \mathrm{ml} / \mathrm{kg})\end{array}$ & $0.30 \pm 0.01^{\mathrm{ab}}$ & $0.30 \pm 0.01^{\mathrm{abc}}$ & $0.31 \pm 0.02^{\mathrm{ab}}$ \\
\hline \multirow{2}{*}{ 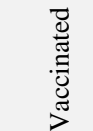 } & $\begin{array}{l}\text { Phytobiotic } \\
(1 \mathrm{ml} / \mathrm{kg})\end{array}$ & $0.29 \pm 0.01^{\mathrm{ab}}$ & $0.30 \pm 0.01^{\mathrm{abc}}$ & $0.29 \pm 0.01^{\mathrm{abc}}$ \\
\hline & $\begin{array}{l}\text { Synobiotic + hytobiotic } \\
(1 \mathrm{ml} / \mathrm{Kg})\end{array}$ & $0.28 \pm 0.01^{\mathrm{b}}$ & $0.27 \pm 0.01^{\mathrm{c}}$ & $0.25 \pm 0.01^{\mathrm{c}}$ \\
\hline
\end{tabular}


a, b \& c: There is no significant difference $(\mathrm{P}>0.05)$ between any two means, within the same column have the same superscript letter.

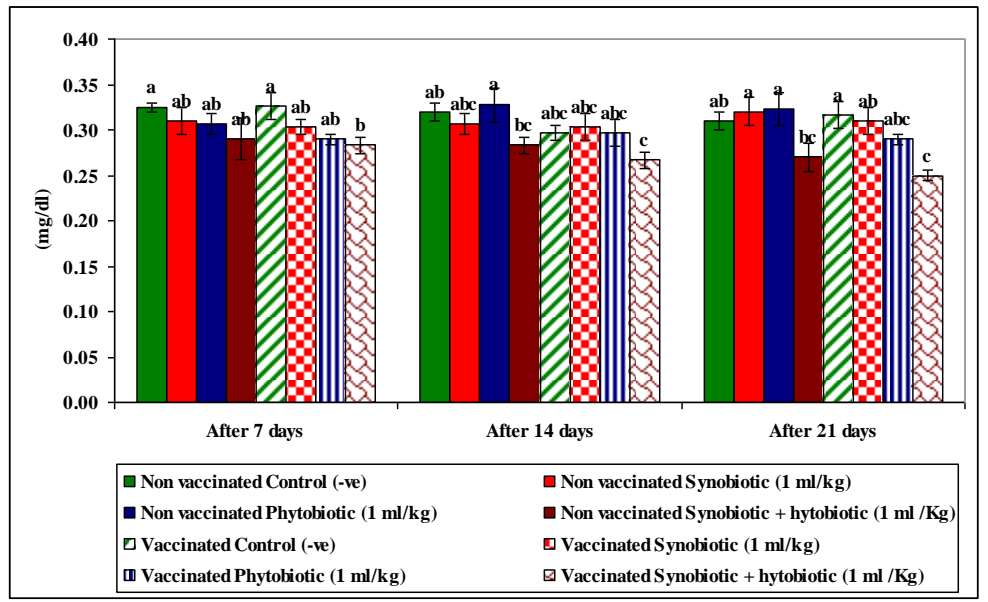

Fig. 2: Creatinine.

\subsection{Effects on hematological parameters}

\subsubsection{The effect on hemoglobin (Hb)}

Therapeutic dose of synobiotic and phyobiotic ( $1 \mathrm{ml} / \mathrm{kg}$ body weight) or mixture of them orally for 3 days on Hb level in both unvaccinated and vaccinated chicken are shown in Table (3) and figure (3). Demonstrated synobiotic ( $1 \mathrm{ml} / \mathrm{kg}$ body weight) in non vaccinate chicken showed decrease of $\mathrm{Hb}$ level at (7 day and 21 day) compared to control group and revealed somewhat increase level at 14 day with unvaccinated chicken, showing decreasing level at all time in vaccinated chicken. Adminisration of phytobiotic ( $1 \mathrm{ml} / \mathrm{kg}$ body weight) orally for 3 days in unvaccinated and vaccinated chicken showed increase of Hb level at (7day,14 day ) compared to control group and slight decrease level at 21 day. Mixture of synobiotic and phytobiotic showed excessive increase in Hb level with both unvaccinated and vaccinated chicken during all periods, while the $\mathrm{Hb}$ level in unvaccinated chicken at 14 day was significant decrease.

Table 3: $\mathrm{Hb}(\mathrm{G} / \mathrm{Dl})($ Mean $\pm \mathrm{SE})$

\begin{tabular}{|c|c|c|c|c|}
\hline \multirow{2}{*}{ Groups } & & \multicolumn{3}{|c|}{ Period after (days) } \\
\hline & & 7 & 14 & 21 \\
\hline \multirow{6}{*}{ 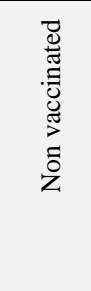 } & Control (-ve) & $10.23 \pm 0.09^{\mathrm{abc}}$ & $10.47 \pm 0.23^{\mathrm{bc}}$ & $11.09 \pm 0.42^{\mathrm{ab}}$ \\
\hline & $\begin{array}{l}\text { Synobiotic } \\
(1 \mathrm{ml} / \mathrm{kg})\end{array}$ & $9.57 \pm 0.21^{\mathrm{c}}$ & $11.04 \pm 0.19^{\mathrm{b}}$ & $11.07 \pm 0.19^{\mathrm{b}}$ \\
\hline & $\begin{array}{l}\text { Phytobiotic } \\
(1 \mathrm{ml} / \mathrm{kg})\end{array}$ & $11.00 \pm 0.58^{\mathrm{a}}$ & $10.66 \pm 0.31^{b}$ & $10.74 \pm 0.22^{\mathrm{b}}$ \\
\hline & Synobiotic + hytobiotic $(1 \mathrm{ml} / \mathrm{Kg})$ & $10.50 \pm 0.41^{\mathrm{abc}}$ & $14.02 \pm 0.27^{\mathrm{a}}$ & $12.19 \pm 0.74^{\mathrm{a}}$ \\
\hline & Control (+ve) & $9.70 \pm 0.31^{\mathrm{bc}}$ & $10.23 \pm 0.09^{\mathrm{bc}}$ & $11.42 \pm 0.29^{\mathrm{ab}}$ \\
\hline & $\begin{array}{l}\text { Synobiotic } \\
(1 \mathrm{ml} / \mathrm{kg})\end{array}$ & $10.00 \pm 0.58^{\mathrm{abc}}$ & $9.68 \pm 0.35^{\mathrm{c}}$ & $9.62 \pm 0.34^{\mathrm{c}}$ \\
\hline \multirow{2}{*}{ 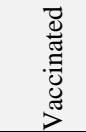 } & $\begin{array}{l}\text { Phytobiotic } \\
(1 \mathrm{ml} / \mathrm{kg})\end{array}$ & $10.63 \pm 0.23^{\mathrm{ab}}$ & $10.28 \pm 0.34^{\mathrm{bc}}$ & $10.41 \pm 0.26^{\mathrm{bc}}$ \\
\hline & $\begin{array}{l}\text { Synobiotic + hytobiotic } \\
(1 \mathrm{ml} / \mathrm{Kg})\end{array}$ & $10.00 \pm 0.18^{\mathrm{abc}}$ & $10.70 \pm 0.39^{\mathrm{b}}$ & $11.06 \pm 0.09^{\mathrm{b}}$ \\
\hline
\end{tabular}

a, b \& c: There is no significant difference $(\mathrm{P}>0.05)$ between any two means, within the same column have the same superscript letter.

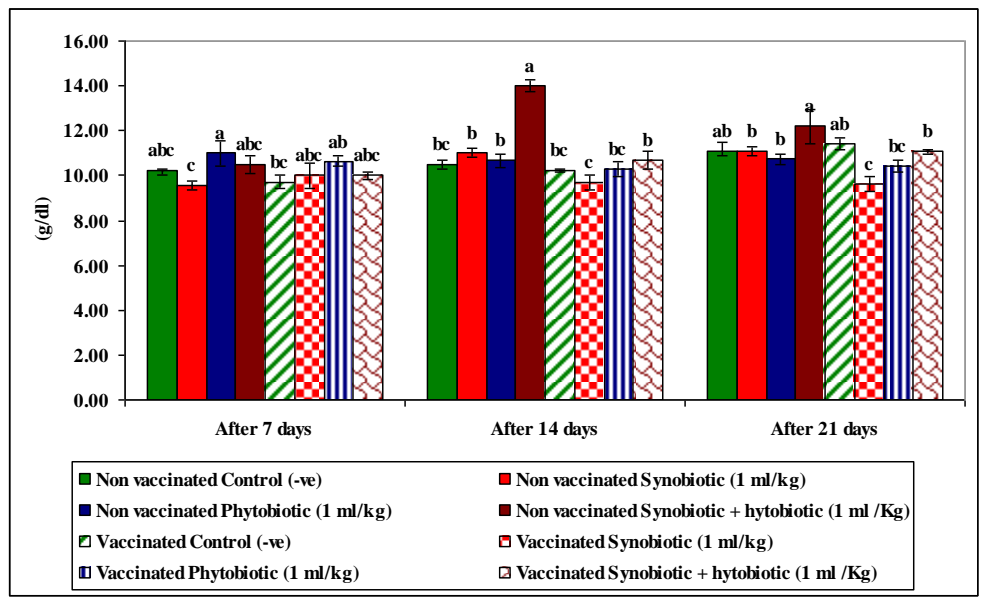

Fig. 3: Hb.

\subsubsection{The effect on RBC}

Table (4) and figure (4) showed the effect of administration synobiotic and phyobiotic and mixture of them in unvaccinated and vaccinated chicken. Therapeutic dose of synobiotic and phyobiotic $(1 \mathrm{ml} / \mathrm{kg}$ body weight) orally for 3 days on RBC showed increase in 
RBC level with unvaccinated and vaccinated chicken at (7 day,14 day,21day) compared to control group, while RBC level showed significant increase at 7 and 14 day with all chicken. The same previous trend was observed with mixture of them during all periods.

Table 4: Rbcs (X106) (Mean \pm SE)

\begin{tabular}{|c|c|c|c|c|}
\hline \multirow[b]{2}{*}{ Groups } & & \multicolumn{3}{|c|}{ Period after (days) } \\
\hline & & 7 & 14 & 21 \\
\hline \multirow{6}{*}{ 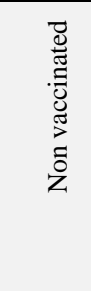 } & Control (-ve) & $1.67 \pm 0.33^{\mathrm{d}}$ & $1.68 \pm 0.33^{\mathrm{c}}$ & $2.90 \pm 0.45^{\mathrm{a}}$ \\
\hline & $\begin{array}{l}\text { Synobiotic } \\
(1 \mathrm{ml} / \mathrm{kg})\end{array}$ & $2.41 \pm 0.29^{\mathrm{bc}}$ & $3.01 \pm 0.02^{\mathrm{ab}}$ & $2.92 \pm 0.07^{\mathrm{a}}$ \\
\hline & $\begin{array}{l}\text { Phytobiotic } \\
(1 \mathrm{ml} / \mathrm{kg})\end{array}$ & $2.58 \pm 0.30^{\mathrm{abc}}$ & $3.27 \pm 0.23^{\mathrm{a}}$ & $3.00 \pm 0.06^{\mathrm{a}}$ \\
\hline & Synobiotic + hytobiotic $(1 \mathrm{ml} / \mathrm{Kg})$ & $3.28 \pm 0.37^{\mathrm{ab}}$ & $2.94 \pm 0.56^{\mathrm{a}}$ & $3.51 \pm 0.39^{\mathrm{a}}$ \\
\hline & Control (+ve) & $2.21 \pm 0.23^{\mathrm{cd}}$ & $2.05 \pm 0.6^{\mathrm{bc}}$ & $2.73 \pm 0.36^{\mathrm{a}}$ \\
\hline & $\begin{array}{l}\text { Synobiotic } \\
(1 \mathrm{ml} / \mathrm{kg})\end{array}$ & $3.33 \pm 0.29^{\mathrm{a}}$ & $2.68 \pm 0.31^{\mathrm{abc}}$ & $2.83 \pm 0.16^{\mathrm{a}}$ \\
\hline \multirow{2}{*}{ 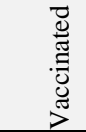 } & $\begin{array}{l}\text { Phytobiotic } \\
(1 \mathrm{ml} / \mathrm{kg})\end{array}$ & $2.46 \pm 0.29^{\mathrm{abcd}}$ & $3.14 \pm 0.3^{\mathrm{ab}}$ & $3.14 \pm 0.30^{\mathrm{a}}$ \\
\hline & $\begin{array}{l}\text { Synobiotic + hytobiotic } \\
(1 \mathrm{ml} / \mathrm{Kg})\end{array}$ & $3.05 \pm 0.19^{\mathrm{abc}}$ & $3.18 \pm 0.41^{\mathrm{ab}}$ & $2.85 \pm 0.25^{\mathrm{a}}$ \\
\hline
\end{tabular}

a, b \& c: There is no significant difference $(\mathrm{P}>0.05)$ between any two means, within the same column have the same superscript letter.

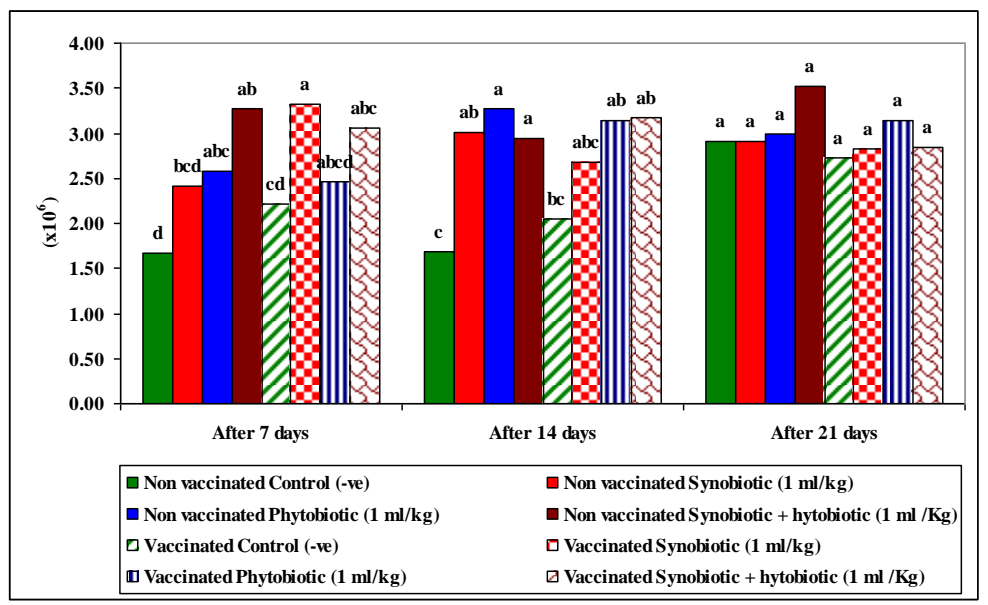

Fig. 4: RBCs.

\subsubsection{The effect on HTC}

The administration synobiotic and phyobiotic and mixture of them in unvaccinated and vaccinated chicken are shown in Table (5) and figure (5). The dose of administration of synobiotic and phyobiotic $(1 \mathrm{ml} / \mathrm{kg}$ body weight) orally for 3 days showed significant decrease in HTC level at (7 day,14 day) with unvaccinated and excessive decrease in vaccinated chicken compared to control group. HTC level with mixture of them in unvaccinated chicken showed significant decrease at 7 day, while at 14 and 21 day tended to increase level compared to control group. The HTC level recorded lower concentration with vaccinated chicken at all times.

Table 5: Haematocrit $(\%)$ (Mean \pm SE)

\begin{tabular}{|c|c|c|c|c|}
\hline \multirow{2}{*}{ Groups } & & \multicolumn{3}{|c|}{ Period after (days) } \\
\hline & & 7 & 14 & 21 \\
\hline \multirow{6}{*}{ 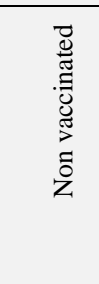 } & Control (-ve) & $36.33 \pm 0.33^{\mathrm{a}}$ & $36.33 \pm 0.33^{\mathrm{ab}}$ & $38.67 \pm 0.33^{\mathrm{b}}$ \\
\hline & $\begin{array}{l}\text { Synobiotic } \\
(1 \mathrm{ml} / \mathrm{kg})\end{array}$ & $33.43 \pm 0.27^{\mathrm{b}}$ & $34.22 \pm 0.32^{\text {cd }}$ & $39.96 \pm 0.06^{\mathrm{ab}}$ \\
\hline & $\begin{array}{l}\text { Phytobiotic } \\
(1 \mathrm{ml} / \mathrm{kg})\end{array}$ & $32.00 \pm 0.58^{\mathrm{c}}$ & $31.00 \pm 0.58^{\mathrm{de}}$ & $37.00 \pm 0.58^{\mathrm{c}}$ \\
\hline & Synobiotic + hytobiotic $(1 \mathrm{ml} / \mathrm{Kg})$ & $30.00 \pm 0.58^{\mathrm{d}}$ & $37.00 \pm 0.58^{\mathrm{a}}$ & $40.67 \pm 0.88^{\mathrm{a}}$ \\
\hline & Control $(+v e)$ & $34.00 \pm 0.58^{b}$ & $35.00 \pm 0.58^{\mathrm{bc}}$ & $39.94 \pm 0.38^{\mathrm{ab}}$ \\
\hline & $\begin{array}{l}\text { Synobiotic } \\
(1 \mathrm{ml} / \mathrm{kg})\end{array}$ & $33.60 \pm 0.31^{\mathrm{b}}$ & $29.00 \pm 0.58^{\mathrm{e}}$ & $31.00 \pm 0.58^{\mathrm{e}}$ \\
\hline \multirow{2}{*}{ 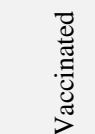 } & $\begin{array}{l}\text { Phytobiotic } \\
(1 \mathrm{ml} / \mathrm{kg})\end{array}$ & $33.67 \pm 0.33^{\mathrm{b}}$ & $33.44 \pm 0.32^{\mathrm{d}}$ & $33.31 \pm 0.35^{\mathrm{d}}$ \\
\hline & $\begin{array}{l}\text { Synobiotic + hytobiotic } \\
(1 \mathrm{ml} / \mathrm{Kg})\end{array}$ & $33.22 \pm 0.37^{\mathrm{bc}}$ & $34.00 \pm 0.58^{\mathrm{cd}}$ & $34.67 \pm 0.33^{\mathrm{d}}$ \\
\hline
\end{tabular}

$\mathrm{a}, \mathrm{b} \& \mathrm{c}$ : There is no significant difference $(\mathrm{P}>0.05)$ between any two means, within the same column have the same superscript letter. 


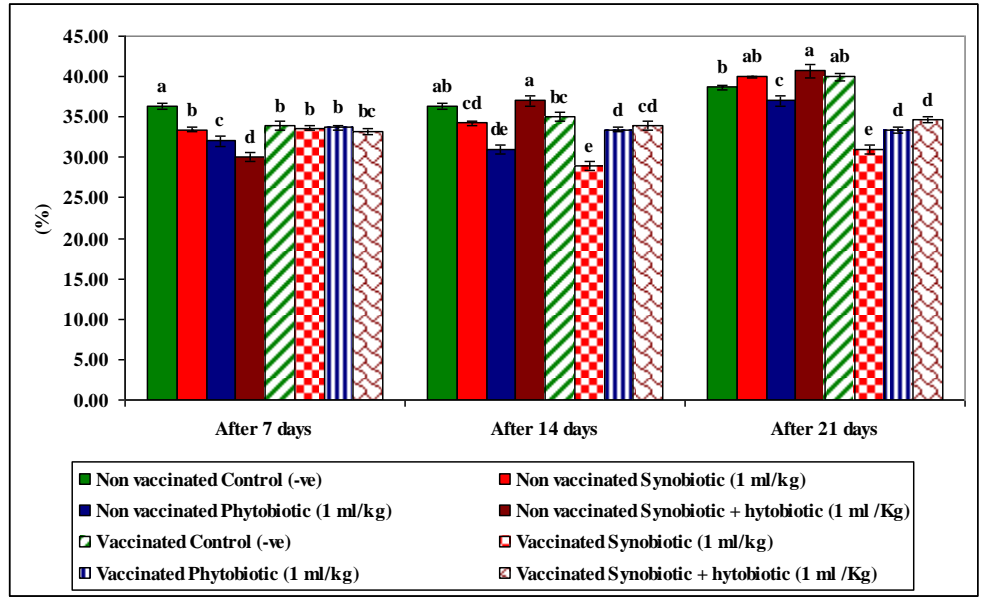

Fig. 5: HCT.

\subsubsection{The effect on MCV}

Table (6) and figure (6) showed the administration synobiotic and phyobiotic and mixture of them in non vaccinated and vaccinated chicken. The dose of administration of synobiotic and phyobiotic ( $1 \mathrm{ml} / \mathrm{kg}$ body weight) orally for 3 days showed significant decrease in MCV level at (7 day,14 day) in unvaccinated and vaccinated chicken. The same trend was observed with phytobiotic. The excessive decrease was shown with mixture of them at 7 1nd 14 day in unvaccinated chicken compared to control group. Mixture of synobiotic and phtobiotic gave slight increase of MCV level in vaccinated chicken during 14 and 21 day compared to control group.

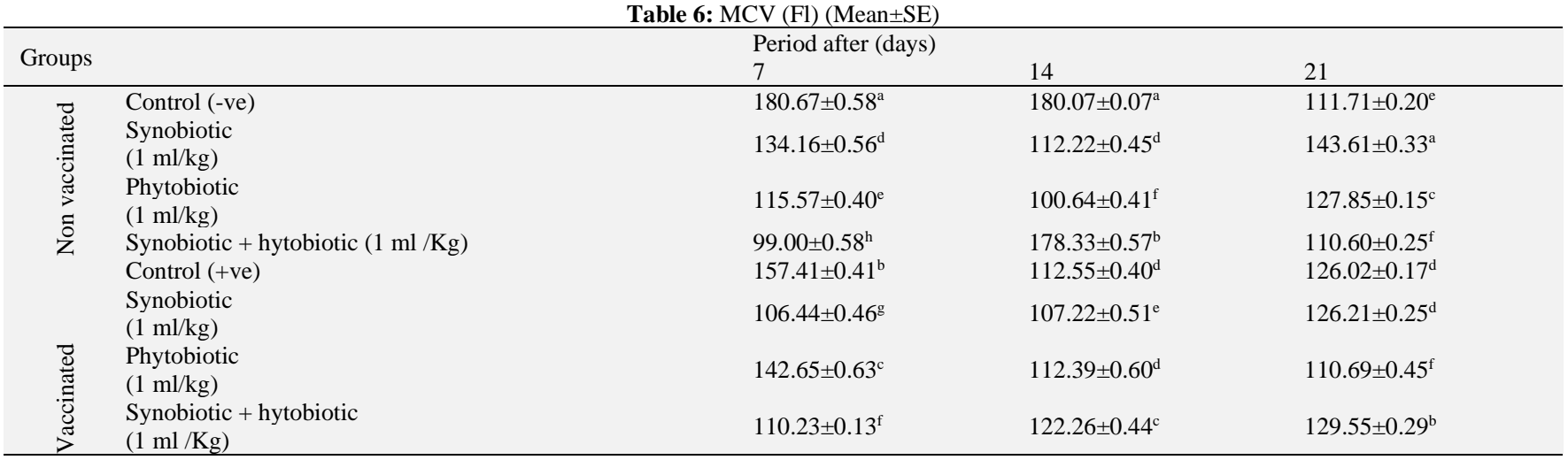

$\mathrm{a}, \mathrm{b} \& \mathrm{c}$ : There is no significant difference $(\mathrm{P}>0.05)$ between any two means, within the same column have the same superscript letter.

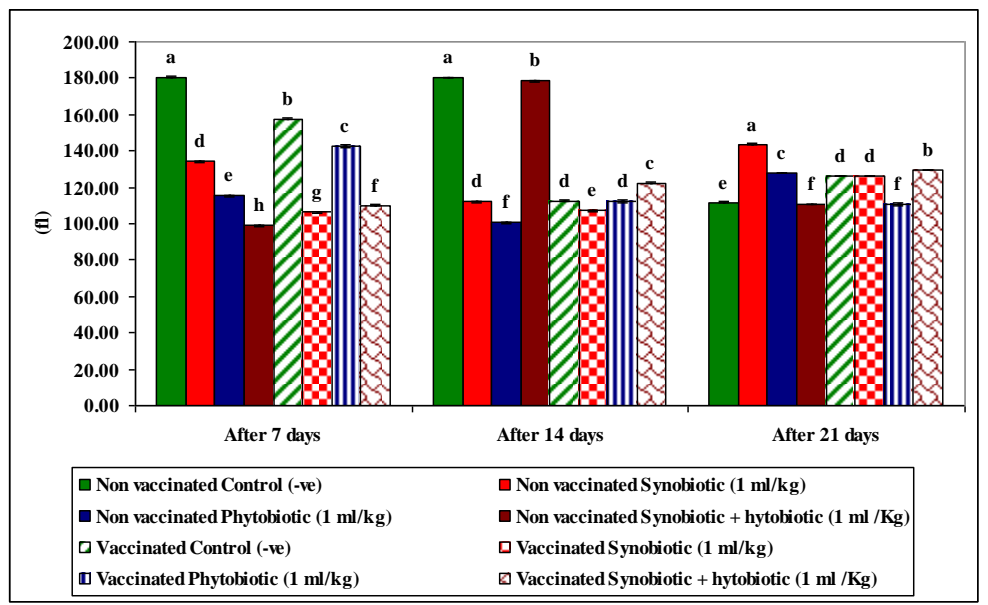

Fig. 6: MCV.

\subsubsection{The effect on WBC}

Administration of synobiotic and phyobiotic and mixture of them in non vaccinated and vaccinated chicken are shown in Table (7) and figure (7). The dose of administration of synobiotic ( $1 \mathrm{ml} / \mathrm{kg}$ body weight) orally for 3 days showed significant increase in WBC level at 7 and 21 day and significant decrease at 14 day. Administration phytobiotic ( $1 \mathrm{ml} / \mathrm{kg}$ body weight) showed significant decrease of WBC level at (7 day and 14 day) and significant increase in WBC level at 21 day in unvaccinated chicken. WBC level in vaccinated chicken appeared to significant decrease at (7 day,21day), while it showed higher significant at 14 day compared to control group. WBC level with mixture of synobiotic and phytobiotic with unvaccinatedchicken showed increase level at all periods, while it was significant higher at 14 day with vaccinated. 


\begin{tabular}{|c|c|c|c|c|}
\hline Groups & & \multicolumn{3}{|c|}{ Period after (days) } \\
\hline \multirow{6}{*}{ 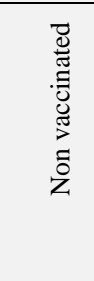 } & Control (-ve) & $76.50 \pm 0.60^{\mathrm{b}}$ & $70.96 \pm 0.06^{\mathrm{a}}$ & $66.36 \pm 0.15^{\mathrm{e}}$ \\
\hline & $\begin{array}{l}\text { Synobiotic } \\
(1 \mathrm{ml} / \mathrm{kg})\end{array}$ & $85.64 \pm 0.24^{\mathrm{a}}$ & $65.76 \pm 0.34^{\mathrm{b}}$ & $69.04 \pm 0.09^{\mathrm{de}}$ \\
\hline & $\begin{array}{l}\text { Phytobiotic } \\
(1 \mathrm{ml} / \mathrm{kg})\end{array}$ & $68.53 \pm 0.42^{\mathrm{c}}$ & $61.33 \pm 0.33^{\mathrm{c}}$ & $71.00 \pm 0.58^{\mathrm{cd}}$ \\
\hline & Synobiotic + hytobiotic $(1 \mathrm{ml} / \mathrm{Kg})$ & $77.47 \pm 0.35^{\mathrm{b}}$ & $71.44 \pm 0.50^{\mathrm{a}}$ & $82.04 \pm 0.10^{\mathrm{b}}$ \\
\hline & Control (+ve) & $70.33 \pm 0.33^{\mathrm{c}}$ & $55.43 \pm 0.48^{\mathrm{d}}$ & $87.00 \pm 0.58^{\mathrm{a}}$ \\
\hline & $\begin{array}{l}\text { Synobiotic } \\
(1 \mathrm{ml} / \mathrm{kg})\end{array}$ & $75.37 \pm 0.32^{\mathrm{b}}$ & $47.67 \pm 0.62^{\mathrm{e}}$ & $88.31 \pm 0.26^{\mathrm{a}}$ \\
\hline \multirow{2}{*}{ 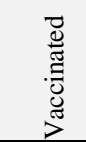 } & $\begin{array}{l}\text { Phytobiotic } \\
(1 \mathrm{ml} / \mathrm{kg})\end{array}$ & $68.36 \pm 0.38^{c}$ & $70.67 \pm 0.88^{a}$ & $82.00 \pm 0.58^{b}$ \\
\hline & $\begin{array}{l}\text { Synobiotic + hytobiotic } \\
(1 \mathrm{ml} / \mathrm{Kg})\end{array}$ & $59.21 \pm 3.41^{\mathrm{d}}$ & $67.21 \pm 0.44^{\mathrm{b}}$ & $74.06 \pm 3.04^{\mathrm{c}}$ \\
\hline
\end{tabular}

$\mathrm{a}, \mathrm{b} \& \mathrm{c}$ : There is no significant difference $(\mathrm{P}>0.05)$ between any two means, within the same column have the same superscript letter.

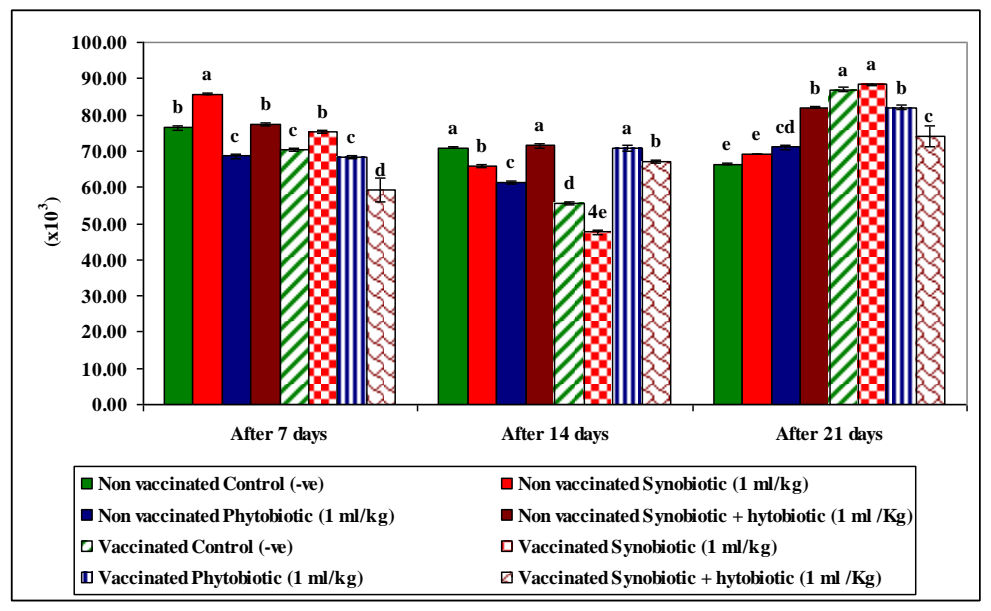

Fig. 7: WBCs.

\subsection{Histological studies}

Microscopic examination of different sections were taken from specimens of kidney. showing normal renal tubules, glomeruli with mild congestion of renal blood vessels figure (9), revealing moderate improvement in histological structure of renal tissue figure (10), showing moderate improvement of kidney lesion figure (11), mild improvement in histological appearance of renal tissue figure (12), showing marked improvement in pathological lesion, except congestion of some renal vessels figure (13).

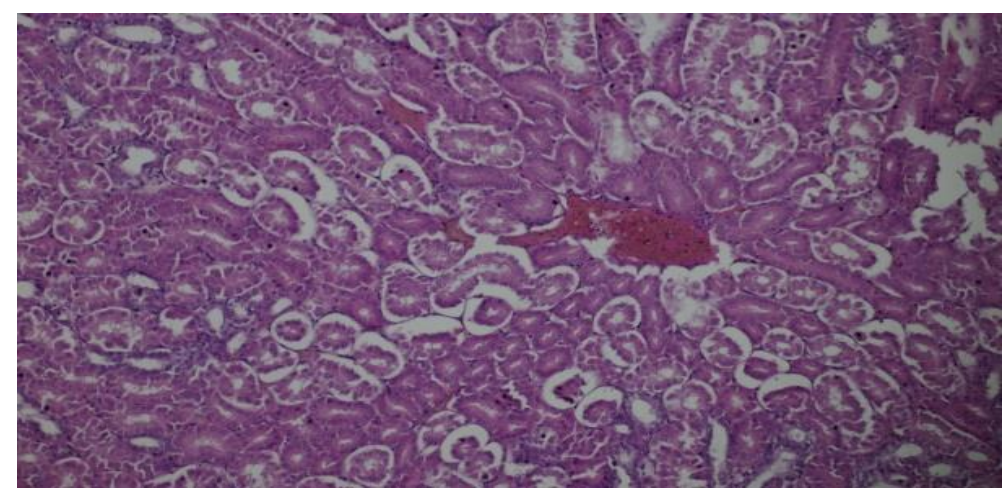

Fig. 9: Showing Normal Renal Tubules, Glomeruli with Mild Congestion of Renal Blood Vessels.

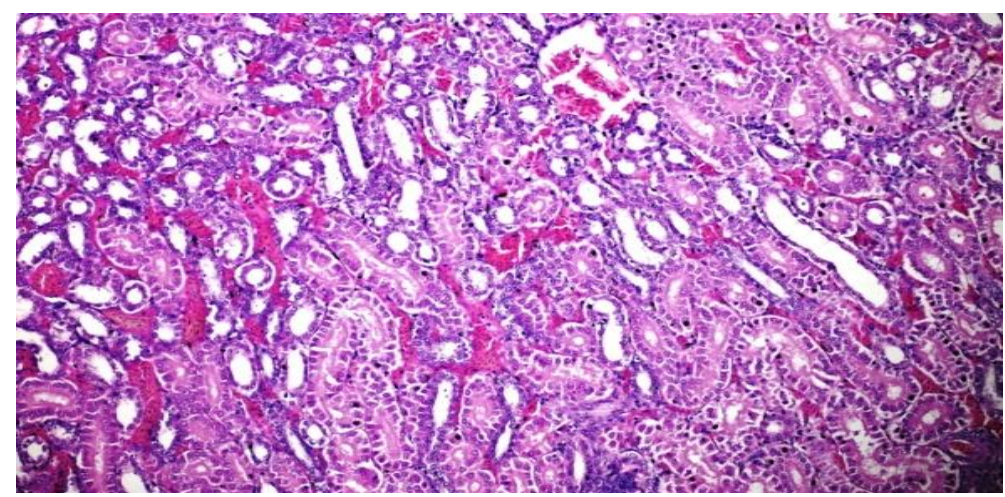

Fig. 10: Showing Moderate Improvement in Histological Structure of Renal Tissue. 


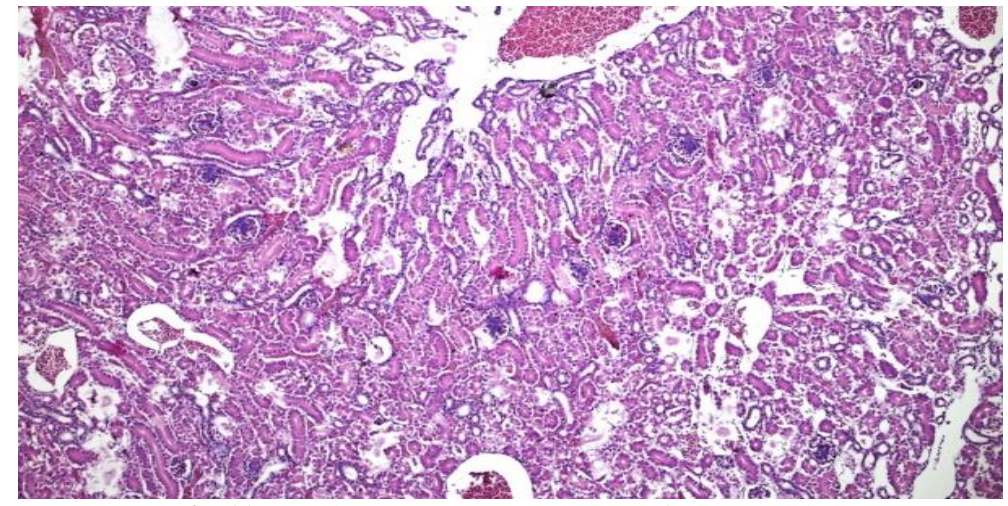

Fig. 11: Showing Moderate Improvement of Kidney Lesion.

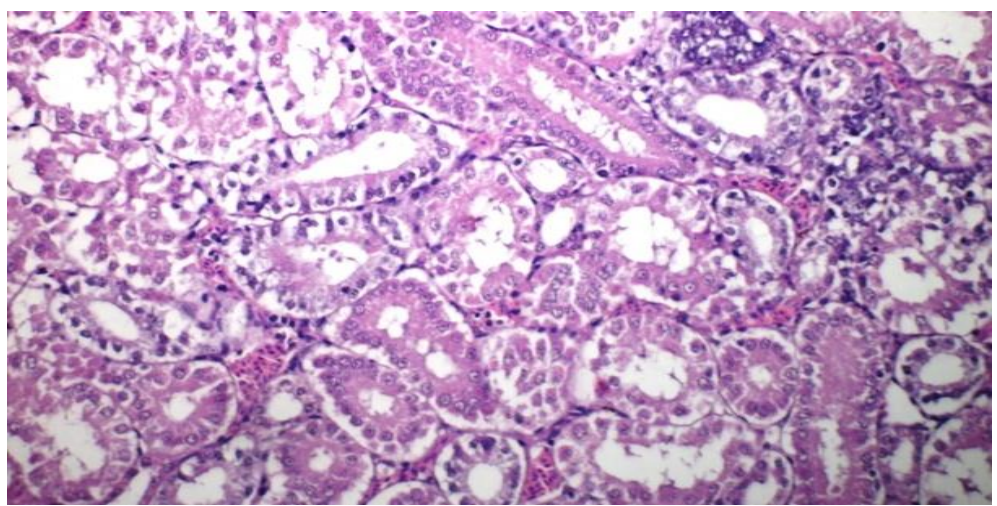

Fig. 12: Revealing Mild Improvement in Histological Appearance of Renal Tissue .

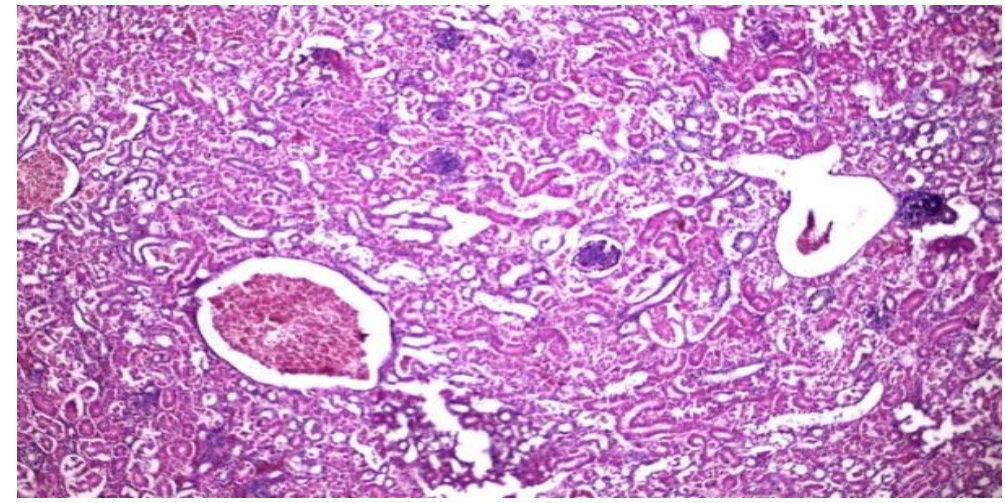

Fig. 13: Showing Marked Improvement in Pathological Lesion, Except Congestion of Some Renal Vessels.

\section{Discussion}

Feed additives include feeding attractants, immunostimulants, prebiotics, probiotics, acidifiers, essential oils, or other inclusions. These additives are usually not required nutrients. Feed attractants enhance the ingestion of feeds. Ashayerizadeh et al (2011) studied the paper effect of dietary supplementation as probiotic and prebiotic on growth indices and serum biochemical parameters of broiler chickens.

Synobiotic Is defined as a mixture of probiotic and prebiotic that beneficially affect the host by improving the survival and activity of beneficial microorganisms in the gut.(Awad et al.; 2008).

Phytobiotic is aphytogenic as feed additive (a mixture of essential oils with thymol and anethole) affected the growth performance, some blood parameters and jejunal morphology in broiler chickens. Aromatic plants, also known as herbs and spices, have been used since antiquity as folk medicine and as preservatives in foods ( Amad et al, 2011).

In order to achieve better results for broilers chicken, various additives can be added to feed, including phytobiotics (phytogenic additives). Phytobiotics protect young broilers' health based on the principles of competitive exclusion and improved usability of nutrients, growth and feed effciency. Sobayo et al, (2013) studied haematological, serum and carcass characteristics of broiler chicken fed graded levels of Garcinia kola (Bitter kola) used as phytobiotic. On the other hand, Sanja et al (2015) studied the effect of synobiotic on growth and antioxidant status of blood in broiler chicken.

kidney is an important organ having not only excretory function but also other functions such as production of the substances that activates a living body, enzymatic reaction, immunization. The kidney is often involved in the development, maintenance and counter regulation of complex electrolyte disturbances ( Heidland et al., 1985).

Uric acid is the primary catabolic product of protein, non protein nitrogen and purines in birds. Birds are uricoletic and produce uric acid not urea as the major nitrogenous end product of metabolism, so blood urea is not effective in renal function test (Harrison and Harrison 1986). Creatinine not a major non protein nitrogen component in avian blood formed during muscle metabolism of creatinine and phosphor creatine and excreted by glomerular filtration. .

In our study there were a significant decrease in uric acid and creatinine level at (7day, 14day and 21day) in unvaccivated and vaccinated chicken. The results were agreement with those reported by Mehr et al., (2014) who observed that the addition of various concentrations 
of clove essential oil, as a natural growth promoter and probiotic resulted in uric acid was significantly decreased in group treated with probiotic. The results were coordinates with that of Abdelrafea et al., (2013). They showed that the addition of Astragalus membranaceus root powder to broiler diets significantly decreased serum creatinine enzymes. Furthermore Erdogan et al.,(2010) studied the effectiveness of phytobiotic as feed supplementation on broilers growth performance, level of creatinine and uric acid in broiler chicken resulted in significant improvement of kidney functions .

Mixture of synobiotic and phytobiotic showed excessive increase in Hb level with both unvaccinated and vaccinated chicken during all periods. Data were agremeent with Jagmohan Singh et al., (2013) who evaluated the effects of Aloe vera on dressing percentage ,hematological and biochemical parameters of broiler chickens, showed significantly higher value of hemoglobin concentration ,also .these results also agremeent with Sobayo et al.,(2013) who studied haematological, serum and carcass characteristics of broiler chicken fed 'graded levels of garcinia kola. Beski et al., (2015) reported that effects of dietary supplementation of probiotic and synbiotic on broiler chickens resulted in a significant increase in the concentration of Hb. Furthermore, results of Syed Muddassar et al.,(2018) were aggrement with the finding of present result. Also, the results were coordinates with that of Fasanmi et al., (2014).

WBC level with mixture of synobiotic and phytobiotic with unvaccinated chicken showed increase level at all periods. These results were agreement with those obtained by Miraghaee et al., (2011) who showed that the addition of Echinacea purpurea Moench extract as feed additive increased white blood count significantly.

\section{Conclusion}

The present study by adminsration of synobiotic and phytobiotic or their mixture lead to significant improvement of growth performance and kidney functions of brolier chicken. Moreover, some mesurments of blood parameters and histological studies were at the normal range with no adverse effects.

\section{Acknowledgment}

Author would like to express her deepest gratitude and sincere thanks to Prof. Dr. Ashraf A.A. EL-Komy, Professor and head of Pharmacology Department, Faculty of Veterinary Medicine, Benha University, for his guidancy and helps and Dr. Enas A. H. Farag Deputy Animal Health Research Institute, Benha Branch, for her continual direction and helps.

\section{References}

[1] Alaeldein M. A.; Mohamed A.M.(2015): Effects of the Dietary Inclusion of a Probiotic, a Prebiotic or their Combinations on the Growth Performance of Broiler Chickens. Brazilian Journal of Poultry Science. . vol.17 .099-104.

[2] Abdelrafea A. E.; Mohammed A. A.; Abdelrahman A.S. and Arafa, M.M. (2013): Influence of Different Levels of Astragalus Root Powder in Broiler Chick Diets on the Physiological and Biochemical Changes. Journal of Applied Sciences Research, 9(3): 2104-2118, 2013 ISSN 1819$544 X$.

[3] Amad, A.A.; Männer, K.; Wendler, K.R.; Neumann, K. and Zentek, J. (2011). Effects of a phytogenic feed additive on growth performance and ileal nutrient digestibility in broiler chickens. Poultry Science, 90: 2811-281. https://doi.org/10.3382/ps.2011-01515.

[4] Ashayerizadeh A.; Dabiri N.; Mirzadeh K. H. M. and Ghorbani M. R. (2011): Paper Effect of dietary supplementation of probiotic and prebiotic on growth indices and serum biochemical parameters of broiler chickens. Journal of Cell and Animal Biology Vol. 5(8), pp. 152-156.

[5] Awad K. Ghareeb S. Abdel-Raheem J. Böhm (2008): Effects of dietary inclusion of probiotic and synbiotic on growth performance, organ weights, and intestinal histomorphology of broiler chickens. Poultry Science, Volume (7): 686-691.

[6] Bancroft, J.D.and Gamble, M. (2008) :"Theory and Practice of Histological Techniques.”' 6th ED., Churchill Livingstone, Elsevier, China. 2008.

[7] Beski S.S.M and Al-Sardary S.Y.T(2015): effects of dietary supplementation of probiotic and synbiotic on broiler chickens hematology and intestinal integrity. International Journal of Poultry Science 14 (1): 31-36. https://doi.org/10.3923/ijps.2015.31.36.

[8] Coshen,R.R.(1967):Anticoagulation, centrifugation time and sample replicate number in micro haematocrite method for avian blood https://doi.org/10.3382/ps.0460214.

[9] Duncan, D.B. (1955): Multiple range and multiple F tests. Biometrics 11: 1-42. https://doi.org/10.2307/3001478.

[10] Erdogan, Z.; Erdogan, S.; Aslantas,O. and Celik,S. (2010) : Effects of dietary supplementation of synbiotics and phytobiotics on performance, caecal coliform population and some oxidant/antioxidant. https://doi.org/10.1111/j.1439-0396.2009.00973.x.

[11] Fasanmi, O.G.; Akanbi, I.O.; Adekunle, I.F.; Oladele-Bukola, M.O.; Makinde, G.E.O.and Fagbohun,F.A. (2014) : Comparing the Phytobiotic effects of feed-grade and liquid Oregostim ${ }^{\circledR}$ on Performance characteristics, Haematology and Serum biochemistry of Finisher broilers. Scholars Journal of Agriculture and Veterinary Sciences 2014; 1(3):137-142.

[12] Folin, O. Z.(1934): Colorimetric determination of creatinine Phys.Chem.268:228. https://doi.org/10.1515/bchm2.1934.228.3-6.268.

[13] Harison, G. J. and Harrison, L.R.(1986): Cited in clinical Avian Medicine and surgery.W.B.Saunders Company. Philadelphia, London, $58: 193-$ 201.

[14] Heidland , A.; Horl, W.H.; Schaefer, R.M.; Teschner, M.; Weipert, J.and Heidbreder, E.(1985): Role of alcohol in clinical nephrology. Klinische Wochenschrift, 63(18):948-958. https://doi.org/10.1007/BF01738150.

[15] Jagmohan Singh.; K. M. Koley.; Khushboo Chandrakar. and Nileshkumar S. Pagrut. (2013) : Effects of Aloe vera on dressing percentage and haemato-biochemidal parameters of broiler chickens . Veterinary World, EISSN: 2231-0916 . https://doi.org/10.14202/vetworld.2013.803-806.

[16] Murarolli V.I; Burbarelli M.; Polycarpo G.I; Ribeiro P.; Moro M.AND Albuquerque R.(2014): Prebiotic, probiotic and symbiotic as alternative to ntibiotics on the Performance and Immune Response of Broiler Chickens. Brazilian Journal of Poultry Science. . vol.16 no.3. https://doi.org/10.1590/1516-635x1603279-284.

[17] Mehr, M.A.; Hassanabadi, A.; Moghaddam, H.N. and Kermanshahi, H. (2014): Supplementation of Clove Essential Oils and Probiotic to the Broiler's Diet on Performance, Carcass Traits and Blood Components. Iranian Journal of Applied Animal Science, 4(1): (117-122).

[18] Miraghaee, S.S.; Heidary, B.; Almasi, H.; shabani, A ; Elahi, M. and Nia, M.H. (2011): The effects of Nigella sativa powder (black seed) and Echinacea purpurea (L.) Moench extract on performance, some blood biochemical and hematological parameters in broiler chickens. African Journal of Biotechnology, 10(82): 19249-19254. https://doi.org/10.5897/AJB11.2891.

[19] Natt, M.P. and Herrick, C.A. (1952): New blood diluents for counting erythrocytes and leucocytes of chickens. Poultry Science Journal, 31:735738. https://doi.org/10.3382/ps.0310735.

[20] Sawsan M. E.; Abdalim F. A.; Mohamed K. M.; Mohamed S. H. E and (2019) Science .Volume 10 (3): 134-140, 2016.

[21] Sanja J. P.; Ljiljana M. K., Nikola M. P.;, Jovanka D. L., Olivera M. Đ.; Bojana M. K.; Ivana S. Č.and Marina V. V.(2015): Effect of synbiotic on growth and antioxidant status of blood in broiler chicken Journal of the Institute of Food Technology in Novi Sad. Volume 42, Issue 2.163-169

[22] SPSS Inc. Released (2009), PASW Statistics for Windows, Version 18.0. Chicago: SPSS Inc. 
[23] Sobayo R.A., Adeyemi O.A. , Oso A.O., Fafiolu A.O., Daramola J.O., Sodipe G., I Ogunade IB , Odetolao O.M.(2013): Haematological, serum and carcass characteristics of broiler chicken fed 'graded levels of Garcinia kola (Bitter kola) used as phytobiotic. Nigerian J. of Animal Production. Vol 40, No 1 https://doi.org/10.51791/njap.v40i1.623.

[24] Syed M.; H.; G., Sitwat Z., Faiz ul-Ha., Saddia G.and Asma A.(2018): Effect of natural growth promoters on immunity, and biochemical and haematological parameters of broiler chickens. Tropical Journal of Pharmaceutical Research. > vol 17, no $4.627-633$ https://doi.org/10.4314/tjpr.v17i4.9.

[25] White, B. A.; Erickson, M. M.; and Stevens, S.G.(1970): Colorimetric determination of uric acid. Chemistry for medical technologists 3rd ed C. V. Mosby Company, saint Louis, U

[26] Wintrobe, M.M. (1967): Colorimeterically Method for Determination Haemoglobin. Medicine Journal 12(5): $287-309$. 